\title{
Role of histological regression grade after two neoadjuvant approaches with or without radiotherapy in locally advanced gastric
} cancer

\author{
Patricia Martin-Romano ${ }^{1}$, Jose J Sola², Juan A Diaz-Gonzalez ${ }^{1}$, Ana Chopitea ${ }^{1}$, Yohana Iragorri ${ }^{1}$, \\ Fernando Martínez-Regueira ${ }^{3}$, Mariano Ponz-Sarvise ${ }^{1}$, Leire Arbea ${ }^{1}$, Jose C Subtil ${ }^{4}$, David Cano ${ }^{5}$, \\ Lucia Ceniceros ${ }^{1}$, Jairo Legaspi ${ }^{1}$, Jose Luis Hernandez ${ }^{3}$ and Javier Rodríguez ${ }^{\star}, 1$ \\ ${ }^{1}$ Department of Oncology, Clínica Universidad de Navarra, Pamplona 31008, Spain; ${ }^{2}$ Department of Pathology, Hospital San \\ Pedro, Logroño 26006, Spain; ${ }^{3}$ Department of Surgical Oncology, Clínica Universidad de Navarra, Pamplona 31008, Spain; \\ ${ }^{4}$ Department of Gastroenterology, Clínica Universidad de Navarra, Pamplona 31008, Spain and ${ }^{5}$ Department of Radiology, Clínica \\ Universidad de Navarra, Pamplona 31008, Spain
}

Background: The degree of histopathological response after neoadjuvant therapy in locally advanced gastric cancer (GC) is a key determinant of patients' long-term outcome. We aimed to assess the pattern of histopathological regression after two neoadjuvant approaches and its impact on survival times.

Methods: Regression grade of the primary tumour (Becker criteria) and the degree of nodal response by a 4-point scale (grades A-D) were assessed. Grade A-true negative lymph nodes (LNs); grade B and C-infiltrated LNs with any or little evidence of nodal response; and grade D—complete pathological response in a previously infiltrated LN. A favourable pathological response was defined as Becker la-b and grade D.

Results: From 2004 to 2014, 80 patients with GC (cT3-4/N + by CT-scan/EUS) were treated with either preoperative chemotherapy $(C h T, n=34)$ or chemoradiation (CRT, $n=46)$. Patients in the CRT group had a higher likelihood of achieving a Becker la-b response (58 vs 32\%, $P=0.001$ ), a grade D nodal regression ( 30 vs $6 \%, P=0.009$ ) and a favourable pathological response ( 23 vs $3 \%$; $P=0.019$ ). Patients with a grade $D$ nodal response had a longer 5-year PFS and OS compared with those with a grade $B$ or $C$ response. Patients with a baseline negative $L N$ status had similar outcomes irrespective of the preoperative therapy received (5-year OS; ChT vs CRT, 58 vs 51\%, P=0.92).

Conclusions: Preoperative chemoradiation increases the likelihood of achieving favourable histopathological features that correlate with a 5 -year OS > 70\% in GC patients.

Although in recent decades both incidence of gastric cancer (GC) and related mortality have decreased, this malignancy still accounts for over $10 \%$ of cancer deaths and is currently the third most frequent cause of cancer-related death worldwide (Torre et al, 2015). Surgery with microscopically negative margins (R0) remains the only potentially curative therapy for patients with localised resectable GC. However, most patients are diagnosed with stage II-III disease and long-term outcomes remain below 30-40\% due to high rates of disease recurrence (Ychou et al, 2011; Takahashi et al, 2013). In this setting, several strategies aimed at increasing

*Correspondence: Dr J Rodríguez; E-mail: jrodriguez@unav.es

Received 25 April 2016; revised 24 June 2016; accepted 19 July 2016; published online 18 August 2016

(c) 2016 Cancer Research UK. All rights reserved 0007-0920/16 
the likelihood of a R0 usually included chemotherapy, radiotherapy or both in the pre or postoperative setting, although the optimal therapeutic sequence has yet to be defined (Macdonald et al, 2001; Cunningham et al, 2006). Although perioperative chemotherapy is one standard of care, preoperative chemoradiotherapy in patients with locally advanced GC is still considered investigational. Nevertheless, several groups have reported their experience with this approach, with encouraging results in terms of R0 resection rates, pathological complete responses and long-term overall survival (OS; Ajani et al, 2004, 2005, 2006; Badgwell et al, 2015).

In this context, a promising line of research is the study of the impact of the degree of histopathological response after neoadjuvant therapy on patient outcomes. It has been suggested that such pathological feature, rather than baseline clinical stage, may predict disease control. The use of preoperative chemoradiation in oesophageal and gastroesophageal junction (GEJ) adenocarcinomas yield higher rates of major pathological responses when compared with those seen with neoadjuvant chemotherapy. Similar findings, although more limited and preliminary, seem true for GC patients (Stahl et al, 2009; Van Hagen et al, 2012). Previous data from our group study suggested a superior regression grade rate after induction chemotherapy followed by chemoradiotherapy in comparison with that seen with chemotherapy alone (DiazGonzalez et al, 2011). However, this analysis included patients with both GC and GEJ cancer, and an in-depth analysis of nodal regression grade was lacking. Given the lack of head-to-head comparisons between these neoadjuvant strategies, the primary aim of this study was to compare the degree of histopathological regressions responses in both, the tumour wall and the lymph nodes, in GC patients treated with neoadjuvant chemotherapy with or without radiotherapy. The secondary aim of this study was to assess whether the regression grade achieved after neoadjuvant therapy may predict long-term outcome.

\section{MATERIALS AND METHODS}

Patient characteristics. From October 2004 to July 2014, patients with clinical stage II-III GC treated at our institution with preoperative therapy followed by surgery were retrospectively reviewed.

Inclusion criteria for neoadjuvant treatment were age from 18 to 75 years, presence of a locally advanced (cT3-4and/or N + ) gastric adenocarcinoma according to the TNM system (American Joint Committee on Cancer, 7th edition), no other primary tumour in the previous 5 years, a good performance status ( $0-1$ according to Eastern Cooperative Oncology Group (ECOG)), and adequate haematological, renal and liver function. Exclusion criteria were patients with a tumour arising in the GEJ (Siewert I-III) and those with non-adenocarcinoma histology, distant metastatic disease (M1), positive peritoneal cytology or peritoneal carcinomatosis, as were those considered unfit for the treatment protocol due to comorbidities.

Preoperative therapy consisted of perioperative chemotherapy alone (ChT group) or induction chemotherapy followed by concurrent chemoradiotherapy (CRT group). A multidisciplinary team that included gastrointestinal surgeons, endoscopists, radiologists, and medical and radiation oncologists evaluated all patients. On the basis of patient's age, ECOG performance status, comorbidities and the clinical stage of the primary tumour, the most suitable therapeutic strategy was recommended. One approach, based on preliminary data from phase II trials (Ajani et al, 2004, 2005, 2006) was to offer preoperative chemoradiation to those patients who were fit, lacked comorbidities and had $\mathrm{N}+$ disease on the baseline workup.
Baseline patient evaluation included clinical examination, blood tests, upper gastrointestinal tract endoscopic ultrasound (EUS) with biopsy, and chest-abdominal computerised tomography-scan (CT-scan) to define the extent of the disease. CT-scan was performed to exclude distant metastases and to evaluate the presence of a gastric mass or gastric thickening and regional lymph nodes ( $\mathrm{N}$ stage). EUS identified tumours penetrating at least the subserosa connective tissue $(\geqslant \mathrm{T} 3)$ along with metastases in regional lymph nodes $(\mathrm{N}+)$ according to previously reported criteria (homogeneous hypoechoic pattern, sharply demarcated margin, rounded contour and size $>10 \mathrm{~mm}$; Catalano et al, 1994). In the presence of suspicious signs of peritoneal disease (ascites or $\geqslant \mathrm{T} 4 \mathrm{a}$ ), an exploratory laparoscopy was accomplished. A CT-scan \pm EUS was performed to assess response after completion of neoadjuvant protocol. In the CRT group, radiological assessment was carried out after completion of both induction chemotherapy and chemoradiation, and before surgery.

Preoperative chemotherapy. All patients from ChT and CRT groups received chemotherapy on an outpatient basis with a pretreatment evaluation that consisted of physical examination and blood tests, treatment-induced adverse effects assessment and a regular clinical follow-up.

Preoperative radiotherapy. For radiotherapy planning, patients were immobilised in the supine position, and a planning CT-scan (slice thickness, 5-10 mm) was performed (Somatron Plus 4; Siemens Oncology Care Systems, Heidelberg, Germany). The target volumes and organs at risk were contoured on each of the axial CT slices in the Helax-TMS treatment planning system (Nucletron Scandinavia, Uppsala, Sweden) or in the ADAC Pinnacle treatment planning system (Philips Radiation Oncology Systems, Fitchburg, WI, USA). Three-dimensional conformal external beam radiotherapy $(93.5 \%)$ or intensity-modulated technique (IMRT; 6.5\%) was used. The clinical target volume included the gross tumour volume along with the entire stomach and the draining locoregional lymph nodes (perigastric, suprapancreatic, coeliac, splenic hilar, porta hepatis and pancreatoduodenal). Conformation and field arrangement ensured that the organs at risk (kidneys, heart, liver and spinal cord) received tolerable doses. Treatment planning followed International Commission on Radiation Units and Measurements recommendations. In general, three fields with 15-MV photons were employed to deliver 45 Gy over 5 weeks with conventional daily fractions of $1.8 \mathrm{~Gy}, 5$ days per week. Seven coplanar, equally spaced beams were applied in a variable number of segments in IMRT plans. All patients received fluoropyrimidine-based concurrent chemotherapy. Patients were evaluated weekly with physical examination, blood test monitoring and therapy-induced toxicity management.

Surgery. Surgery was scheduled 4 to 6 weeks after the completion of the neoadjuvant treatment. Location and extent of the primary tumour determined the type of surgery to be used, which included either distal-subtotal gastrectomy or total gastrectomy as indicated by the multidisciplinary board criteria. Spleen preservation was performed. An attempt was made to perform a D2-type nodal dissection in all patients.

Toxicity. Toxicity during ChT and CRT was graded according to the National Cancer Institute Common Toxicity Criteria v4.0.

Pathological response assessment. A gastrointestinal pathologist (JJS) performed the pathological examination of all the surgical specimens. TNM status was assessed postoperatively in accordance with the 7th edition of the AJCC cancer staging. A complete pathological response (pCR) was considered when no evidence of residual tumour was found in the surgical specimen (ypT0 ypN0). Histological regression grade of the primary tumour was assessed according to the Becker criteria: grade la (complete tumour 
regression), grade $1 \mathrm{~b}$ ( $<10 \%$ of vital tumour tissue), grade 2 (residual tumour per tumour bed of 10-50\%) and grade 3 ( $>50 \%$ of viable tumour remaining; Becker et al, 2003). Becker grades $\mathrm{Ia}$ and $\mathrm{Ib}$ were considered to be a major pathological response.

The degree of nodal response was evaluated using a four-point scale derived from the Miller and Payne grading system for breast cancer (Smith et al, 2002). The scale describes the pathological features of lymph nodes after neoadjuvant therapy as follows: Grade A-true negative lymph nodes with no evidence of preoperative therapy effect; Grade B-infiltrated lymph nodes with no evidence of any effect of preoperative therapy; Grade $\mathrm{C}$ infiltrated lymph nodes with evidence of some degree of histological regression due to preoperative therapy; Grade Dcomplete pathological response in a previously infiltrated node. A favourable pathological response was defined as a combination of Becker grade $1 \mathrm{a}-\mathrm{b}$ response plus grade $\mathrm{D}$ nodal regression. Downstaging was defined as a reduction in the pathological $\mathrm{T}$ and/ or $\mathrm{N}$ stage (ypTNM) compared with baseline clinical staging (cTNM).

Statistics. Differences in the proportions were calculated with a $\chi^{2}$-test with Fisher's correction when needed by the number of cases. Patient pathological responses were cross-tabulated by age, gender, ECOG performance status, tumour location, tumour histological grading, Lauren classification, baseline $\mathrm{T}$ and $\mathrm{N}$ stages, type of neoadjuvant treatment group (ChT vs CRT) and the type of systemic chemotherapy regimen. Any association between each of these factors and response was examined using a $\chi^{2}$-test or Fisher's exact test.

Progression-free survival (PFS) was calculated from the date of diagnosis to the date of progression (local and/or distant), death or last contact. OS was determined from diagnosis to time of death or last contact when still alive. Survival times were analysed by treatment group and subgroups of pathological tumour and/or nodal response.

The statistical analysis was performed using SPSS statistical software (version 20.0 for Windows; SPSS, Chicago, IL, USA). All statistical tests were conducted at a two-sided significance level of 0.05 .

Written informed consent was obtained in all cases. The institutional review board approved this retrospective analysis.

\section{RESULTS}

Patient groups. Patients' baseline characteristics are described in Table 1. The two groups were well balanced, except for sex and EUS-nodal stage (more men, uN + and AJCC stage III patients in the CRT group).

Preoperative therapy. In the $\mathrm{ChT}$ group, 34 patients were evaluated. Chemotherapy encompassed trial protocols of oxaliplatin-fluoropyrimidines $(n=11)$ and off-protocol treatment with taxane-based therapy including docetaxel-oxaliplatin-capecitabine $(n=15)$ and paclitaxel-cisplatin-5FU $(n=8)$. The median number of cycles administered was 3 (range 2-6). After the end of ChT,

\section{Table 1. Baseline characteristics}

\begin{tabular}{|c|c|c|c|c|}
\hline Characteristic & CT group (n (\%)) & CRT group (n (\%)) & Global series (n \%)) & $P$ value (CT vs CRT) \\
\hline Number of patients & 34 & 46 & 80 & - \\
\hline Age (median) & 64.5 & 61.5 & $62(36-83)$ & 0.46 \\
\hline $\begin{array}{c}\text { ECOG } \\
0 \\
1\end{array}$ & $\begin{array}{c}3(8.8) \\
31(91.2)\end{array}$ & $\begin{array}{c}2(4.3) \\
44(95.7)\end{array}$ & $\begin{array}{c}5(5) \\
75(95)\end{array}$ & 0.67 \\
\hline $\begin{array}{l}\text { Location } \\
\text { Antrum } \\
\text { Body } \\
\text { Pylorus }\end{array}$ & $\begin{array}{c}17(50) \\
17(50) \\
-\end{array}$ & $\begin{array}{c}25(54.3) \\
19(41.4) \\
2(4.3)\end{array}$ & $\begin{aligned} 42 & (52.5) \\
36 & (45) \\
2 & (2.5)\end{aligned}$ & 0.39 \\
\hline $\begin{array}{l}\text { Histologic grade } \\
\text { Well differentiated } \\
\text { Moderately differentiated } \\
\text { Poorly differentiated }\end{array}$ & $\begin{array}{c}1(3) \\
13(38.2) \\
20(58.8)\end{array}$ & $\begin{aligned} & 1(2.2) \\
& 17(37) \\
& 28(60.8)\end{aligned}$ & $\begin{aligned} & 2(2.5) \\
30 & (37.5) \\
48 & (60)\end{aligned}$ & 0.82 \\
\hline $\begin{array}{l}\text { Baseline EUS-T stage } \\
\text { T2 } \\
\text { T3 } \\
\text { T4 } \\
\text { T4a } \\
\text { T4b }\end{array}$ & $\begin{aligned} 1 & (2.9) \\
23 & (67.6) \\
10 & (31.4) \\
9 & (26.5) \\
1 & (2.9)\end{aligned}$ & $\begin{array}{c}-1 \\
36(78.3) \\
10(21.7) \\
6(13) \\
4(8.7)\end{array}$ & $\begin{aligned} 1 & (1.3) \\
59 & (73.7) \\
20 & (25) \\
15 & (18.7) \\
5 & (6.3)\end{aligned}$ & 0.94 \\
\hline $\begin{array}{l}\text { Baseline EUS-N stage } \\
\text { NO } \\
\mathrm{N}+\end{array}$ & $\begin{array}{l}15(44.1) \\
19(55.9)\end{array}$ & $\begin{array}{r}5(10.9) \\
41(89.1)\end{array}$ & $\begin{array}{l}20(25) \\
60(75)\end{array}$ & 0.001 \\
\hline Exploratory laparoscopy & - & $20(43.5 \%)$ & $20(25 \%)$ & $<0.001$ \\
\hline $\begin{array}{l}\text { AJCC stage } \\
\| \\
\|\|\end{array}$ & $\begin{array}{l}16(47) \\
18(53)\end{array}$ & $\begin{array}{r}5(10.9) \\
41(89.1)\end{array}$ & $\begin{array}{l}21(26.3) \\
59(73.7)\end{array}$ & $<0.001$ \\
\hline
\end{tabular}


responses were assessed by CT-scan (CR 9\%; PR 29\%; SD 24\%) and/or EUS (response 74\%; no response 14\%; NA 12\%).

In the CRT group, 46 patients were assessed. Induction chemotherapy included trial protocols of oxaliplatin-fluoropyrimidines $(n=3)$, ECF $(n=1)$, CF $(n=1)$ and off-protocol treatment with taxane-based therapy including docetaxel-oxaliplatin-capecitabine $(n=19)$, FLOT $(n=12)$ and paclitaxel-cisplatin$5 F U(n=10)$. The median number of induction chemotherapy cycles was 3 (range 2-5). During CRT, mean dose of EBRT administered was $44.5 \mathrm{~Gy}$ (range, 37.8-46), and the median treatment length was 34 days (range, 29-43). At the completion of the whole-neoadjuvant approach, responses were evaluated with CT-scan (CR 11\%; PR 46\%; SD 37\%; PD 2\%; NA 4\%) and/or EUS (response 67\%; no response 16\%; NA 17\%).

Toxicity. During CRT, 13 patients (28\%) required admission to hospital: 2 patients (4\%) due to asthenia; 3 patients $(7 \%)$ for asthenia and anorexia; 4 patients (9\%) for asthenia, anorexia and mucositis; 3 patients (7\%) for asthenia, anorexia and gastritis; and 1 patient (2\%) for cardiac insufficiency. Eight patients admitted to hospital required total parenteral nutrition. No grade IV toxicities were observed. Concurrent chemotherapy was completed in 31 patients (67\%). Six patients (13\%) needed a break during radiotherapy. Four patients (9\%) did not receive the initially planned radiotherapy due to toxicity. Treatment-induced adverse events are listed in Supplementary Table 1.

Surgery. In all, 77 patients (96\%) underwent surgery. On an intend-to-treat basis an R0 resection was achieved in 73 patients
(91\%). Three patients (4\%) were not operated, all of them from the CRT group. One patient developed systemic relapse in the preoperative staging. The other two patients achieved radiological stable disease but unresectability criteria were observed in the operating room. A R0 resection rate above $90 \%$ was achieved in both groups, with no differences between them $(P=0.81)$. In the CRT group, 1 patient (2\%) died from a suture dehiscence at day 7 from surgery and 2 patients (7\%) experienced operative complications: small bowel perforation and mesocolon bleeding. In the ChT group, 1 patient (3\%) presented an anastomotic bleeding (Supplementary Table 2).

Pathological analysis. Pathological examination of the surgical specimens (Table 2) revealed a pCR in 9 patients: 3 patients (9\%) from the ChT group, and 6 patients (14\%) from the CRT group $(P=0.92)$. Patients receiving CRT had a higher likelihood of achieving a major pathological response (Becker 1a-b; 58 vs 32\%; $P=0.001$ ). A favourable pathological response (Becker $1 \mathrm{a}-\mathrm{b}$ plus grade $\mathrm{D}$ nodal regression) was noted in 11 patients: $1(3 \%)$ in the ChT group and $10(23 \%)$ in the CRT group $(P=0.019)$.

No significant differences were found in the proportion of tumour-free lymph nodes in either treatment group (ypN + : 50\% in the ChT group vs $58 \%$ in the CRT group; $P=0.52$ ). However, grade $\mathrm{D}$ nodal regression was more frequently observed in the CRT group (6 vs 30\%; $P=0.009$ ). Forty patients (52\%) achieved a Becker $1 \mathrm{a}-\mathrm{b}$ regression grade and/or a grade $\mathrm{D}$ nodal regression: $35 \%$ in the ChT group and $65 \%$ in the CRT group $(P=0.009)$.

\begin{tabular}{|c|c|c|c|}
\hline & CT group $(n=34(\%))$ & CRT group $(n=43(\%))$ & $P$ value (CT vs CRT) \\
\hline $\begin{array}{l}\text { AJCC pT stage } \\
\text { PT0 } \\
\text { PT1 } \\
\text { PT2 } \\
\text { PT3 } \\
\text { PT4a } \\
\text { PT4b }\end{array}$ & $\begin{aligned} 3 & (8.8) \\
4 & (11.8) \\
6 & (17.7) \\
17 & (50) \\
3 & (8.8) \\
1 & (2.9)\end{aligned}$ & $\begin{aligned} 6 & (13.9) \\
2 & (4.7) \\
11 & (25.6) \\
17 & (39.5) \\
5 & (11.6) \\
& 2(4.7)\end{aligned}$ & 0.92 \\
\hline $\begin{array}{l}\text { AJCC pN stage } \\
\text { pNO } \\
\text { pN }+\end{array}$ & $\begin{array}{l}17(50) \\
17(50)\end{array}$ & $\begin{array}{l}25(58.1) \\
18(41.9)\end{array}$ & 0.84 \\
\hline $\begin{array}{l}\text { Tumour regression grade } \\
\text { 1a } \\
1 \mathrm{~b} \\
2 \\
3\end{array}$ & $\begin{array}{c}3(8.8) \\
8(23.5) \\
8(23.5) \\
15(44.2)\end{array}$ & $\begin{aligned} 6 & (13.9) \\
19 & (44.2) \\
17 & (39.5) \\
1 & (2.4)\end{aligned}$ & $0.001^{a}$ \\
\hline $\begin{array}{l}\text { Nodal regression grade } \\
\text { A } \\
\text { B } \\
\text { C } \\
\text { D }\end{array}$ & $\begin{aligned} 17 & (50) \\
7 & (20.6) \\
8 & (23.5) \\
2 & (5.9)\end{aligned}$ & $\begin{aligned} 12 & (27.9) \\
6 & (14) \\
12 & (27.9) \\
13 & (30.2)\end{aligned}$ & $0.005^{\mathrm{b}}$ \\
\hline RO resection & $32(94.1)$ & $41(95.3)$ & 0.81 \\
\hline Favourable pathological response $^{c}$ & $1(2.9)$ & $10(23.3)$ & 0.019 \\
\hline Tumour and/or nodal pathological response ${ }^{d}$ & $12(35.3)$ & $28(65.1)$ & 0.009 \\
\hline $\begin{array}{l}\text { AJCC pathological stage } \\
|-| \mid \\
|| \mid\end{array}$ & $\begin{array}{r}26(76.5) \\
8(23.5)\end{array}$ & $\begin{array}{l}32(74.4) \\
11(25.6)\end{array}$ & 0.84 \\
\hline $\begin{array}{l}\text { TN downstaging } \\
\text { Yes } \\
\text { No }\end{array}$ & $\begin{array}{l}23(67.6) \\
11(32.4) \\
\end{array}$ & $\begin{array}{l}33(76.7) \\
10(23.3) \\
\end{array}$ & 0.37 \\
\hline
\end{tabular}


A downstaging in resected patients was observed in 56 patients (73\%). Overall, downstaging was similar in both treatment arms $(P=0.37)$, as was the final AJCC pathological stage $(P=0.84)$.

Prognostic factors. Patients' characteristics (age, gender and ECOG performance status), tumour characteristics (histologic classification, baseline clinical $\mathrm{T}$ and $\mathrm{N}$ stage), and treatmentrelated characteristics (ChT vs CRT, and type of chemotherapy regimen) were analysed in order to identify potential predictors of achieving a favourable pathological response. As shown in Table 3, the use of preoperative CRT was the only statistically significant variable (23 vs 3\%; $P=0.019)$.

Patients' long-term outcome. After a median follow-up of 66 months (range, 5-105 months), median PFS was 48 months and median OS was 59 months. The 5-year actuarial PFS and OS rates were 49 and $48 \%$, respectively. Six patients died without evidence of disease relapse during the follow-up period; the causes were a postoperative complication after surgery of the stomach (1 patient), a bowel perforation after 14 months of follow-up (1 patient), a second tumour (1 patient), a traumatic head injury (1 patient) and traffic accident ( 2 patients). There were no statistically significant differences between the two neoadjuvant strategies neither in median PFS (CT vs CRT; NR vs 48 months, $P=0.56$ ) nor in median OS (ChT vs CRT; 51 vs 71 months; $P=0.24$, Figure 1 ).

Survival times were also calculated according to pathological features. Five-year actuarial PFS was significantly longer in ypN0 patients compared to ypN + patients (70 vs 31\%, $P=0.01$ ), as was the 5-year actuarial OS (58 vs 40\%; $P=0.03$ ). However, 5-year actuarial PFS for Becker 1a-b was similar to Becker 2-3 (55 vs 47\%; $P=0.39$ ) along with 5-year OS (55 vs $46 \% ; P=0.49$ ).

In a subgroup analysis, we excluded patients with a grade A nodal regression (truly negative $\mathrm{LN}$ from baseline) to rule out the impact of the degree of pathological nodal response in patients' outcome. Patients with a complete nodal response (grade D) showed an improvement in 5-year OS (64 vs 40\%; $P=0.066$ ) and PFS (79 vs 31\%; $P=0.043$ ) compared with that seen in patients with partial or no nodal response (grades B and C; Figure 2). There was a trend towards higher 5-year actuarial PFS and OS rates for patients with favourable pathological response (PFS 75 vs 38\%, $P=0.31$; OS 60 vs $31 \%, P=0.17$; Figure 2). No survival differences were observed between patients with grades $\mathrm{A}$ or $\mathrm{D}$ nodal regression in either 5-year PFS (64 vs 79\%; $P=0.48$ ) or 5-year OS (54 vs 64\%; $P=0.59$ ).

Long-term outcome of patients with Becker $1 \mathrm{a}-\mathrm{b}$ and either grade A or D nodal regression was similar (5-year PFS 76 and $72 \%$, respectively); therefore, we decided to evaluate them jointly, for statistical purposes. Patients achieving a Becker $1 \mathrm{a}-\mathrm{b}$ response and either grade $\mathrm{A}$ or $\mathrm{D}$ nodal regression experienced a survival advantage in terms of 5-year PFS (75 vs 41\%; $P=0.025$ ) and OS ( 66 vs $42 \% ; P=0.011$ ) when compared with that seen in the rest of the patients (Figure 2). There were no survival differences in patients with grade A nodal response regardless of the neoadjuvant treatment received in either 5-year PFS (ChT 53\%; CRT 80\%; $P=0.45$ ) or 5 -year OS (ChT $58 \%$; CRT $51 \%, P=0.92$; Figure 3 ).

Five-year actuarial PFS was significantly longer in patients with a pathological downstaging (79 vs $41 \% ; P=0.027$ ). A statistical trend was also found for these patients in terms of 5-year OS (73 vs $41 \% ; P=0.051)$. In addition, pathological stage I-II patients had a longer 5-year PFS and OS rates than stage III patients (PFS 63 vs $23 \% ; P=0.003$; OS 60 vs $28 \%, P=0.019$ ).

\section{DISCUSSION}

Preoperative chemotherapy in the management of GC patients is a well-accepted approach that has correlated with an improved

\begin{tabular}{|c|c|c|c|}
\hline Covariate & $n=77(\%)$ & $\begin{array}{l}\text { Favourable pathological } \\
\text { response }(n=11(\%))\end{array}$ & $P$-value \\
\hline \multicolumn{4}{|l|}{ Gender } \\
\hline $\begin{array}{l}\text { Male } \\
\text { Female }\end{array}$ & $\begin{array}{l}50(64.9) \\
27(35.1)\end{array}$ & $\begin{array}{l}6(7.8) \\
5(6.5)\end{array}$ & 0.425 \\
\hline \multicolumn{4}{|l|}{ ECOG } \\
\hline $\begin{array}{l}0 \\
1\end{array}$ & $\begin{array}{c}5(6.5) \\
72(93.5)\end{array}$ & $\begin{array}{c}1(1.3) \\
10(12.9)\end{array}$ & 0.548 \\
\hline \multicolumn{4}{|c|}{ Lauren histological classification } \\
\hline $\begin{array}{l}\text { Diffuse } \\
\text { Intestinal }\end{array}$ & $\begin{array}{l}44(57.1) \\
33(42.9)\end{array}$ & $\begin{array}{l}7(9.1) \\
4(5.2)\end{array}$ & 0.749 \\
\hline \multicolumn{4}{|c|}{ Baseline EUS-T stage } \\
\hline $\begin{array}{l}\text { T2 } \\
\text { T3 } \\
\text { T4a-b }\end{array}$ & $\begin{array}{c}1(1.3) \\
57(74) \\
19(24.7)\end{array}$ & $\begin{array}{c}\overline{-} \\
10(12.9) \\
1(1.3)\end{array}$ & 0.262 \\
\hline \multicolumn{4}{|c|}{ Baseline EUS-N stage } \\
\hline $\begin{array}{l}\mathrm{N} 0 \\
\mathrm{~N}+\end{array}$ & $\begin{array}{l}19(24.7) \\
58(75.3)\end{array}$ & $11 \overline{(14.3)}$ & 0.056 \\
\hline \multicolumn{4}{|c|}{ Baseline AJCC stage } \\
\hline $\begin{array}{l}\text { I-II } \\
\text { III }\end{array}$ & $\begin{array}{l}20(26) \\
57(74)\end{array}$ & $11 \overline{(14.3)}$ & 0.057 \\
\hline \multicolumn{4}{|c|}{ Preoperative treatment } \\
\hline $\begin{array}{l}\text { CT } \\
\text { CRT }\end{array}$ & $\begin{array}{l}34(44.2) \\
43(55.8)\end{array}$ & $\begin{array}{c}1(1.3) \\
10(12.9)\end{array}$ & 0.019 \\
\hline \multicolumn{4}{|l|}{$\mathrm{CT}$ regimen } \\
\hline $\begin{array}{l}\text { Taxane based } \\
\text { Non-taxane based }\end{array}$ & $\begin{array}{l}61(79.2) \\
16(20.8)\end{array}$ & $11 \overline{(14.3)}$ & 0.107 \\
\hline
\end{tabular}


A

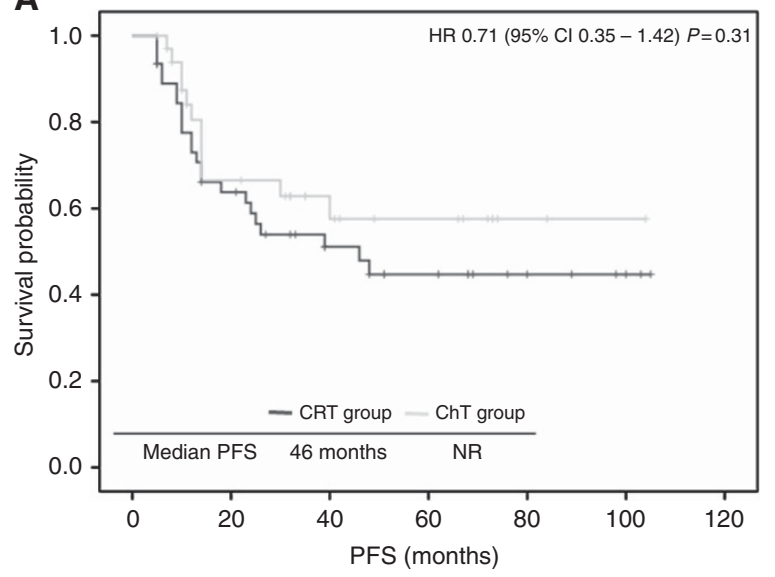

B

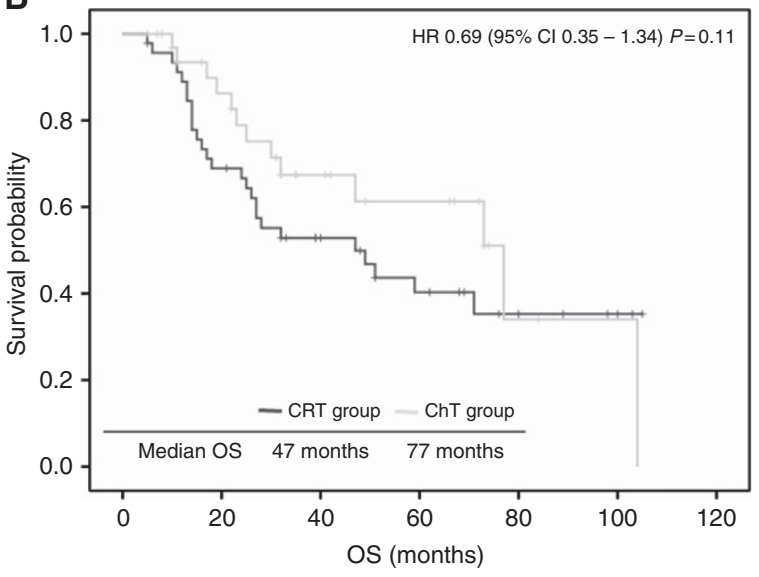

Figure 1. PFS (A) and OS (B) according to preoperative treatment from the global series $(n=80)$.

5-year OS compared with that seen with surgery alone. Conversely, the use of preoperative chemoradiation is currently considered investigational in GC, with only scarce phase II data are available. Potential benefits of neoadjuvant CRT include its ability to increase the rate of $\mathrm{R} 0$ resections and major pathological responses (Ajani et al, 2004, 2005, 2006). In the present analysis, we aimed to evaluate the patterns of pathological response in locally advanced GC patients who received either chemotherapy (ChT group) or induction chemotherapy followed by chemoradiotherapy (CRT group) prior to surgery. The impact of the pathological response on patients' long-term outcome was also analysed.

According to our results, after surgery on an intent-to-treat basis, an $\mathrm{R} 0$ resection rate $>90 \%$ was achieved in both groups (ChT and CRT), which is in line with other studies using these neoadjuvant approaches. These results were particularly promising in the CRT group, even though almost $90 \%$ of the patients were EUS-N,$+ 22 \%$ had T4 tumours and $\sim 70 \%$ had a poorly differentiated histology. Nevertheless, these data should be viewed with caution, given its retrospective exploratory nature and the limited number of patients analysed.

The prognostic relevance of tumour downstaging in GC patients treated with a neoadjuvant approach has been described (Davies et al, 2014), and a pathological complete response has consistently been associated with excellent long-term outcomes (Badgwell et al, 2015). The prognostic role of the grade of histological regression within the tumour wall, however, is more controversial, with some reports suggesting that this variable has no effect on patients' disease-specific survival (Mansour et al, 2007; Fujitani et al, 2012).

Several attempts have been made to evaluate the degree of pathological remission in upper GI malignancies, but no grading system has been developed to date to quantify the magnitude of therapy-induced nodal regression in GC patients (Becker et al, 2003, 2011). This seems an important task, since some reports have described the independent prognostic significance of the degree of tumour response within the lymph nodes (Ott et al, 2011). The grading system used in our work, adapted from Smith et al (2002) in breast cancer, may facilitate the histologic assessment of this residual disease. Grade A and D responses were observed in ypN0 patients, with a mandatory clarification: the pathological features of grade $\mathrm{D}$ patients indicate positive lymph nodes at diagnosis. Indeed, after patients with grade A nodal regression were excluded, 5-year PFS and OS estimates were significantly longer for patients with grade $\mathrm{D}$ responses than for those with grade $\mathrm{B}$ or $\mathrm{C}$ nodal responses (PFS $P=0.066$; OS $P=0.043$ ). This suggests that not only nodal response but also the quality of that response correlates with patients' outcome. Results from our study revealed a higher rate of responsive lymph nodes in the CRT group, which is consistent with the ability of radiation to achieve nodal sterilisation (Macdonald et al, 2001).

No survival differences in either PFS or OS were observed between patients with grades $\mathrm{A}$ or $\mathrm{D}$ nodal regression. These data reinforce the idea of the favourable prognostic impact for ypN0 patients, either because patients have truly negative lymph nodes before the start of therapy or because preoperative therapy achieved a pathologically complete nodal response. The 5-year OS in this subgroup is remarkable and makes ypN0 a good surrogate marker for long-term outcome.

The use of chemoradiation significantly increased the likelihood of achieving a pathological tumour response graded Becker $1 \mathrm{a}-\mathrm{b}$ (58 vs 32\%; $P=0.001$ ); these data support our previous results (Diaz-Gonzalez et al, 2011). Chemoradiation was also correlated with a five-fold increase in the probability of accomplishing a grade $\mathrm{D}$ nodal regression (30 vs 6\%; $P=0.005$ ), and these two features correlated with a 5 -year OS above $70 \%$. Nevertheless, the addition of preoperative radiotherapy did not uniformly result in a survival advantage in our patient population. This fact may be partly attributable to the unbalanced baseline characteristics. Patients in the CRT group had larger tumours and more frequent nodal involvement, as reflected by the percentage of grade A nodal regression in both groups (28vs 50\%, $P=0.005$ ). In addition, postoperative lymph node status and the degree of nodal regression are not the only prognostic indicators in GC patients. The ratio of positive to total lymph nodes harvested, the presence of nodal micrometastasis, perineural or vascular invasion (Mansour et al, 2007; Kutlu et al, 2015) and the new molecular classification and alterations in relevant signalling pathways are all factors that warrant consideration in larger prospective trials (Cancer Genome Atlas Research Network, 2014; Cui et al, 2015).

One of the major issues of concern with the use of preoperative radiation therapy is toxicity. Although treatment compliance was high and no grade 4 toxicities were recorded, almost $30 \%$ of our patients required readmission due to CRT-induced toxicity. Strict eligibility and intensive clinical follow-up seem mandatory in an attempt to improve tolerance and to balance toxic effects and efficacy. On the other hand, rates of morbidity and mortality in the CRT group were 7 and 2\%, respectively. Radiation field or dose administered in these patients were consistent to recommended constrains and thus, these complications were unexpected and may not be related to the radiation therapy. Indeed, morbidity and mortality rates of $7-47 \%$ and $3-10 \%$, respectively, have been described with preoperative strategies (Ajani et al, 2004, 2005, 2006; Cunningham et al, 2006; Stahl et al, 2009; Ychou et al, 2011).

Another argument in favour of more precisely quantifying the amount of residual tumour within LNs is the possibility of 
A

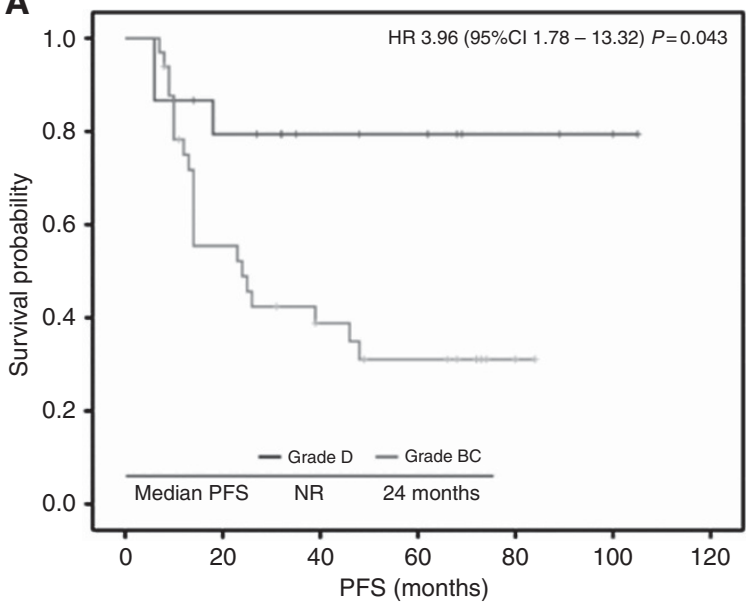

C

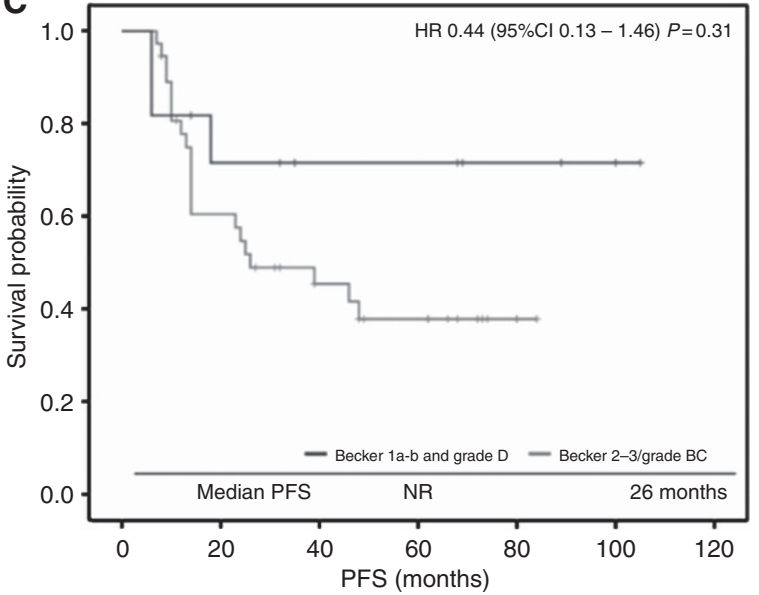

E

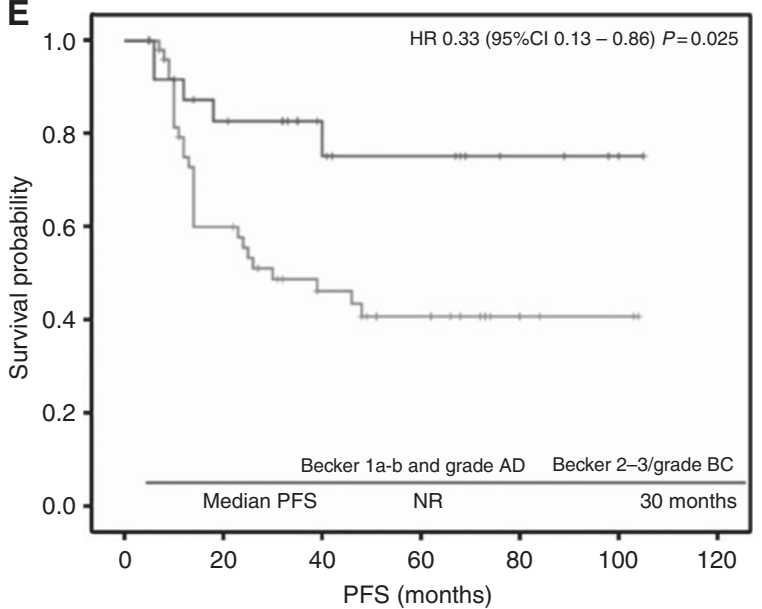

B

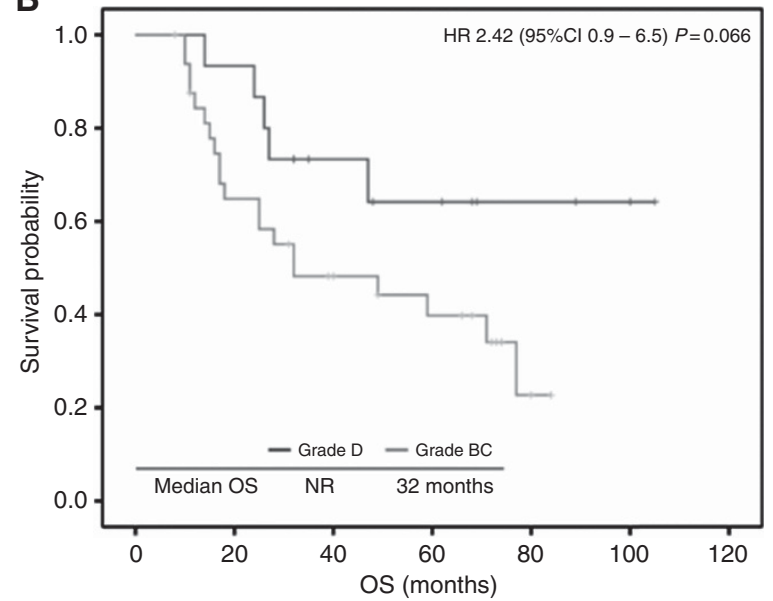

D

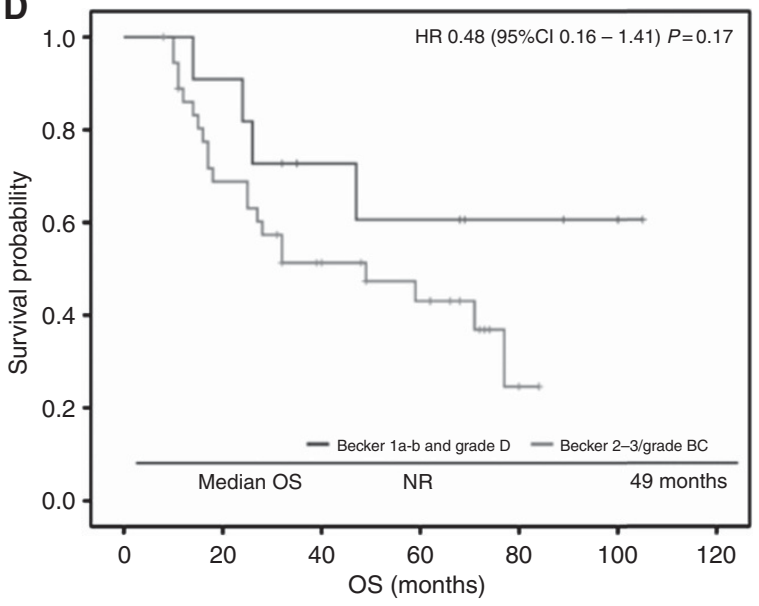

$\mathbf{F}$

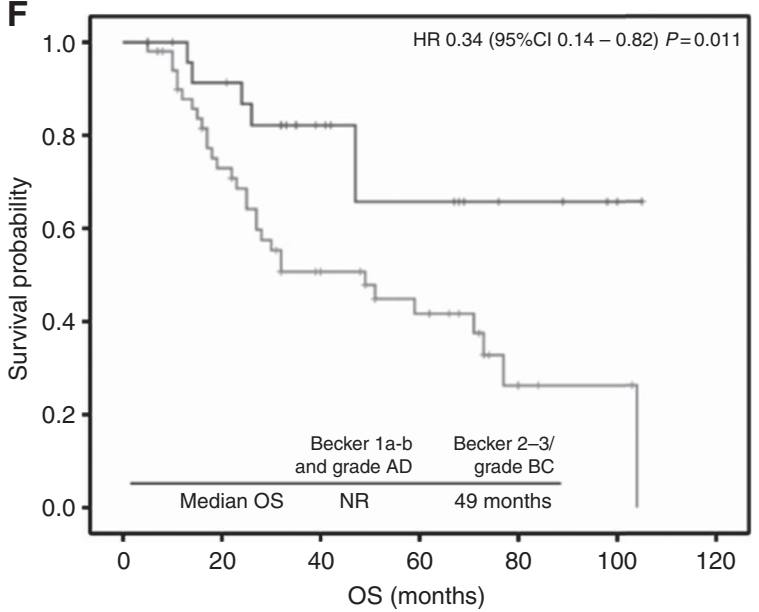

Figure 2. Survival according to pathological responses. PFS (A) and OS (B) according to nodal response (excluding grade A patients; $n=48$ ). PFS (C) and OS (D) according to favourable pathological response (Becker 1a-b and grade D, excluding grade $A$ patients; $n=48)$. PFS (E) and OS (F) according to tumour and nodal response (Becker $1 a-b$ and grade $A$ and $D$ patients; $n=77$ ).

identifying a subgroup of truly negative LNs from baseline, whose clinical outcome would be excellent irrespective of the preoperative therapy administered. Indeed, according to our results, patients with a grade A nodal response had similar survival outcomes with ChT and CRT (PFS $P=0.45$; OS $P=0.92$ ). This may point to a limited value of adding radiation therapy in the neoadjuvant strategy of those patients with a truly negative lymph node status. On the other hand, radiation increases the likelihood of achieving a grade D nodal regression, a known indicator of long-term outcome. Accurate preoperative assessment of the nodal stage seems then to be a major challenge in the attempt to provide a tailored management plan for these patients. One recent study reported that clinical response classified by a combination of CT-scan and EUS correlated with prognosis in GEJ patients treated with neoadjuvant chemotherapy and interestingly that preoperative clinical and histopathological responses were also strongly correlated (Blank et al, 2015). However, the value of these image modalities seems limited in patients receiving chemoradiotherapy, given the difficulty in discriminating between residual tumour and post-radiation changes. Moreover, several authors 
A

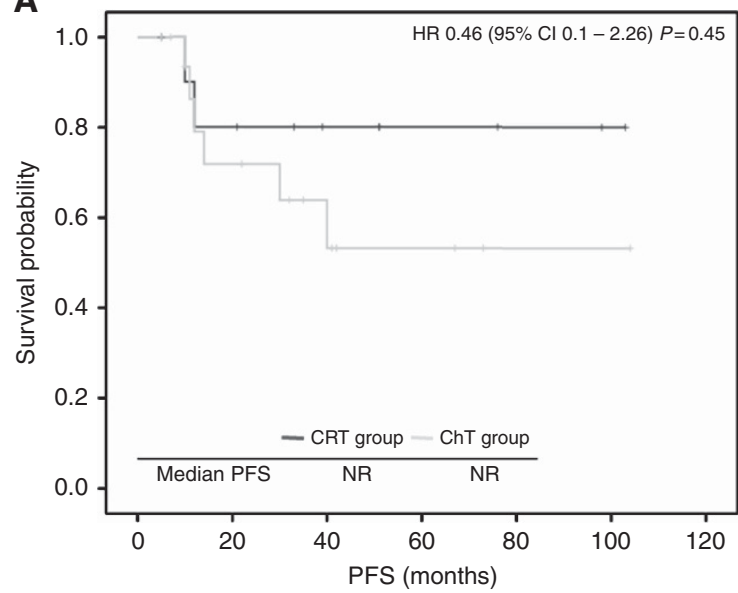

B

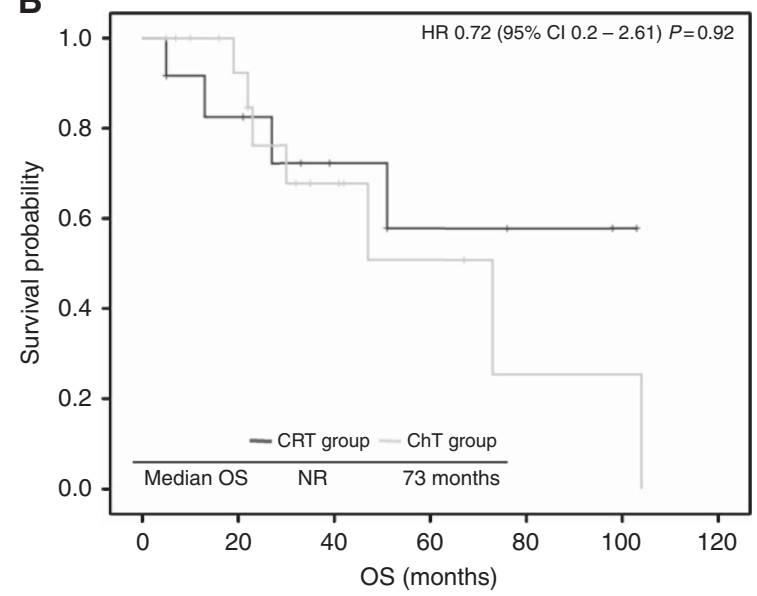

Figure 3. PFS (A) and OS (B) by treatment group in patients with nodal response grade $A(n=29)$.

have found a poor correlation between clinical evaluation of the lymph nodes and tumour regression grade (Kurokawa et al, 2014; Kinoshita et al, 2015). Whether alternative radiological evaluations, such as the combination of EUS and laparoscopic ultrasonography might increase the current accuracy deserves further research.

The main limitations of the present study include its retrospective nature, the small number of patients and the fact that it was conducted at a single institution, so results should be taken with caution. Nevertheless, in the absence of prospective randomised studies, our results suggest that the degree of pathological regression may be a good surrogate of preoperative treatment efficacy. Moreover, long-term survival seems to be influenced by tumour and nodal regression grade. Further prospective studies are required to identify patients who will benefit from preoperative chemoradiation.

\section{CONCLUSIONS}

In conclusion, induction chemotherapy followed by chemoradiation in the preoperative setting in GC patients seems to increase the likelihood of achieving favourable histopathological features, which might eventually impact long-term outcome. Further research in order to establish predictive factors to individualise adjunct therapies to surgery is warranted.

\section{ACKNOWLEDGEMENTS}

We thank all the patients who participated in the study and their families.

\section{CONFLICT OF INTEREST}

The authors declare no conflict of interest.

\section{REFERENCES}

Ajani JA, Mansfield PF, Crane CH, Wu TT, Lunagomez S, Lynch PM, Janjan N, Feig B, Faust J, Yao JC, Nivers R, Morris J, Pisters PW (2005) Paclitaxel-based chemoradiotherapy in localized gastric carcinoma: degree of pathologic response and not clinical parameters dictated patient outcome. J Clin Oncol 23: 1237-1244.
Ajani JA, Mansfield PF, Janjan N, Morris J, Pisters PW, Lynch PM, Feig B, Myerson R, Nivers R, Cohen DS, Gunderson LL (2004) Multi-institutional trial of preoperative chemoradiotherapy in patients with potentially resectable gastric carcinoma. J Clin Oncol 22: 2774-2780.

Ajani JA, Winter K, Okawara GS, Donohue JH, Pisters PW, Crane CH, Greskovich JF, Anne PR, Bradley JD, Willett C, Rich TA (2006) Phase II trial of preoperative chemoradiation in patients with localized gastric adenocarcinoma (RTOG 9904): quality of combined modality therapy and pathologic response. J Clin Oncol 24: 3953-3958.

Badgwell B, Blum M, Estrella J, Chiang Y-J, Das P, Matamoros A, Fournier K, Mansfield P, Ajani J (2015) Predictors of survival in patients with resectable gastric cancer treated with preoperative chemoradiation therapy and gastrectomy. J Am Coll Surg 221: 83-90.

Becker K, Langer R, Reim D, Novotny A, Meyer zum Buschenfelde C, Engel J, Friess H, Hofler H (2011) Significance of histopathological tumor regression after neoadjuvant chemotherapy in gastric adenocarcinomas: a summary of 480 cases. Ann Surg 253: 934-939.

Becker K, Mueller JD, Schulmacher C, Ott K, Fink U, Busch R, Bottcher K, Siewert JR, Hofler H (2003) Histomorphology and grading of regression in gastric carcinoma treated with neoadjuvant chemotherapy. Cancer 98: $1521-1530$.

Blank S, Lordick F, Bader F, Burian M, Dobritz M, Grenacher L, Becker K, Weichert W, Langer R, Sisic L, Stange A, Jager D, Buchler M, Bruckner T, Siewert J, Ott K (2015) Post-therapeutic response evaluation by a combination of endoscopy and CT scan in esophagogastric adenocarcinoma after chemotherapy: better than its reputation. Gastric Cancer 18: 314-325.

Cancer Genome Atlas Research Network (2014) Comprehensive molecular characterization of gastric adenocarcinoma. Nature 513: 202-209.

Catalano MF, Sivak MV, Rice T, Gragg La, Van Dam J (1994) Endosonographic features predictive of lymph node metastasis. Gastrointest Endosc 40: 442-446.

Cui J, Yin Y, Ma Q, Wang G, Olman V, Zhang Y, Chou WC, Hong CS, Zhang C, Cao S, Mao X, Li Y, Qin S, Zhao S, Jiang J, Hastings P, Li F, Xu Y (2015) Comprehensive characterization of the genomic alterations in human gastric cancer. Int J Cancer 137: 86-95.

Cunningham D, Allum WH, Stenning SP, Thompson JN, Van de Velde CJ, Nicolson M, Scarffe JH, Lofts FJ, Falk SJ, Iveson TJ, Smith DB, Langley RE, Verma M, Weeden S, Chua YJ, Participants MT (2006) Perioperative chemotherapy versus surgery alone for resectable gastroesophageal cancer. N Engl J Med 355: 11-20.

Davies AR, Gossage JA, Zylstra J, Mattsson F, Lagergren J, Maisey N, Smyth EC, Cunningham D, Allum WH, Mason RC (2014) Tumor stage after neoadjuvant chemotherapy determines survival after surgery for adenocarcinoma of the esophagus and esophagogastric junction. J Clin Oncol 32: 2983-2990.

Diaz-Gonzalez JA, Rodriguez J, Hernandez-Lizoain JL, Ciervide R, Gaztanaga M, San Miguel I, Arbea L, Aristu JJ, Chopitea A, MartinezRegueira F, Valenti V, Garcia-Foncillas J, Martinez-Monge R, Sola JJ 
(2011) Patterns of response after preoperative treatment in gastric cancer. Int J Radiat Oncol Biol Phys 80: 698-704.

Fujitani K, Mano M, Hirao M, Kodama Y, Tsujinaka T (2012) Posttherapy nodal status, not graded histologic response, predicts survival after neoadjuvant chemotherapy for advanced gastric cancer. Ann Surg Oncol 19: 1936-1943.

Kinoshita O, Ichikawa D, Ichijo Y, Komatsu S, Okamoto K, Kishimoto M, Yanagisawa A, Otsuji E (2015) Histological evaluation for chemotherapeutic responses of metastatic lymph nodes in gastric cancer. World J Gastroenterol 21: 13500-13506.

Kurokawa Y, Shibata T, Sasako M, Sano T, Tsuburaya A, Iwasaki Y, Fukuda H (2014) Validity of response assessment criteria in neoadjuvant chemotherapy for gastric cancer (JCOG0507-A). Gastric Cancer 17: 514-521.

Kutlu OC, Watchell M, Dissanaike S (2015) Metastatic lymph node ratio successfully predicts prognosis in western gastric cancer patients. Surg Oncol 24: 84-88.

Macdonald JS, Smalley SR, Benedetti J, Hundahl SA, Estes NC, Stemmermann GN, Haller DG, Ajani JA, Gunderson LL, Jessup JM, Martenson JA (2001) Chemoradiotherapy after surgery compared with surgery alone for adenocarcinoma of the stomach or gastroesophageal junction. $N$ Engl $J$ Med 345: 725-730.

Mansour JC, Tang L, Shah M, Bentrem D, Klimstra DS, Gonen M, Kelsen DP, Brennan MF, Coit DG (2007) Does graded histologic response after neoadjuvant chemotherapy predict survival for completely resected gastric cancer? Ann Surg Oncol 14: 3412-3418.

Ott K, Lordick F, Blank S, Buchler M (2011) Gastric cancer: surgery in 2011. Langenbecks Arch Surg 396: 743-758.

Smith IC, Heys SD, Hutcheon AW, Miller ID, Payne S, Gilbert FJ, Ah-See AK, Eremin O, Walker LG, Sarkar TK, Eggleton SP, Ogston KN (2002)
Neoadjuvant chemotherapy in breast cancer: significantly enhanced response with docetaxel. J Clin Oncol 20: 1456-1466.

Stahl M, Walz MK, Stuschke M, Lehmann N, Meyer HJ, Riera-Knorrenschild J, Langer P, Engenhart-Cabillic R, Bitzer M, Konigsrainer A, Budach W, Wilke H (2009) Phase III comparison of preoperative chemotherapy compared with chemoradiotherapy in patients with locally advanced adenocarcinoma of the esophagogastric junction. J Clin Oncol 27: 851-856.

Takahashi T, Saikawa Y, Kitagawa Y (2013) Gastric cancer: current status of diagnosis and treatment. Cancers 5: 48-63.

Torre LA, Bray F, Siegel RL, Ferlay J, Lortet-Tieulent J, Jemal A (2015) Global cancer statistics, 2012. CA Cancer J Clin 65: 87-108.

Van Hagen P, Hulshof MC, van Lanschot JJ, Steyerberg EW, van Berge Henegouwen MI, Wijnhoven BP, Richel DJ, Nieuwenhuijzen GA, Hospers GA, Bonenkamp JJ, Cuesta MA, Blaisse RJ, Busch OR, ten Kate FJ, Creemers GJ, Punt CJ, Plukker JT, Verheul HM, Spillenaar Bilgen EJ, van Dekken H, van der Sangen MJ, Rozema T, Biermann K, Beukema JC, Piet AH, van Rij CM, Reinders JG, Tilanus HW, van der Gaast A (2012) Preoperative chemoradiotherapy for esophageal or junctional cancer. N Engl J Med 366: 2074-2084.

Ychou M, Boige V, Pignon JP, Conroy T, Bouche O, Lebreton G, Ducourtieux M, Bedenne L, Fabre JM, Saint-Aubert B, Geneve J, Lasser P, Rougier P (2011) Perioperative chemotherapy compared with surgery alone for resectable gastroesophageal adenocarcinoma: an FNCLCC and FFCD multicenter phase III trial. J Clin Oncol 29: 1715-1721.

This work is published under the standard license to publish agreement. After 12 months the work will become freely available and the license terms will switch to a Creative Commons AttributionNonCommercial-Share Alike 4.0 Unported License.

Supplementary Information accompanies this paper on British Journal of Cancer website (http://www.nature.com/bjc) 